\title{
Tunable thin film bulk acoustic wave resonators with improved $\boldsymbol{Q}$-factor
}

\author{
A. Vorobiev ${ }^{\text {a) }}$ and S. Gevorgian \\ Department of Microtechnology and Nanoscience, Chalmers University of Technology, \\ SE-41296 Gothenburg, Sweden
}

(Received 30 April 2010; accepted 9 May 2010; published online 28 May 2010)

\begin{abstract}
The tunable solidly mounted $\mathrm{Ba}_{0.25} \mathrm{Sr}_{0.75} \mathrm{TiO}_{3}$ (BSTO) thin film bulk acoustic wave resonators (TFBARs) with improved $Q$-factor are fabricated and characterized. The BSTO films are grown by magnetron sputtering at temperature $600{ }^{\circ} \mathrm{C}$ and extremely low sputter gas pressure 2 mTorr using on-axis configuration. The measured TFBARs $Q$-factor is more than 250 and mechanical $Q$-factor is more than 350 at $5 \mathrm{GHz}$ resonance frequency. The improvement in the $Q$-factor is associated with reduction in the BSTO film grain misorientation. The latter is responsible for generation of shear waves leaking through the Bragg reflector and corresponding acoustic loss. (C) 2010 American Institute of Physics. [doi:10.1063/1.3441413]
\end{abstract}

The electrically tunable thin film bulk acoustic wave resonators (TFBARs) are emerging microwave devices utilizing electric field induced piezoelectric effect in paraelectric phase ferroelectrics $\left[\mathrm{Ba}_{\mathrm{x}} \mathrm{Sr}_{1-\mathrm{x}} \mathrm{TiO}_{3}\right.$ (BSTO), for example]. ${ }^{1} \quad$ They enable development of advanced reconfigurable/adaptable circuit architectures (e.g., agile front ends of microwave transceivers). However, the practical applications are hindered by relatively low acoustic $Q$-factor of the tunable TFBARs. Simulations show that $Q f$ product of a TFBAR resonator used in a ladder filter should be more than $2000 \mathrm{GHz}^{2}$ For comparison, the nontunable TFBARs utilizing AlN films reveal average $Q f=3000 \mathrm{GHz}{ }^{2}$ The $Q f$ of the best reported tunable BSTO TFBARs are in the range $400-600 \mathrm{GHz} .^{1,3-5}$ At the same time, extrapolation of $\mathrm{SrTiO}_{3}$ single crystal measurements gives $Q f$ $\approx 6000 \mathrm{GHz}{ }^{6,7}$ This indicates that acoustic quality of the tunable BSTO TFBARs is currently limited by extrinsic loss mechanisms and there is a room for its substantial improvement. In the solidly mounted resonator (SMR) TFBARs there are two main extrinsic loss mechanisms as follows: (i) electrical loss and (ii) leaking waves. ${ }^{2}$ In this paper, we demonstrate that the $Q f$ product of the tunable BSTO SMR TFBARs can be improved, if the BSTO films are fabricated at proper conditions. We associate the improvement with reduction in the BSTO film microstructure imperfections responsible for generation of leaking waves.

In this work, TFBAR test structures, Fig. 1, are based on silicon substrates with resistivity $20 \mathrm{k} \Omega \mathrm{cm}$. The Bragg reflector $\lambda / 4 \mathrm{SiO}_{2} / \mathrm{W}$ layers and the Pt bottom electrode with $\mathrm{W}$ adhesion layer (not shown in the Fig. 1) are deposited by magnetron sputtering. The BSTO films are deposited by magnetron sputtering of the $\mathrm{Ba}_{0.25} \mathrm{Sr}_{0.75} \mathrm{TiO}_{3}$ target in on-axis configuration at $10 \mathrm{~cm}$ of the substrate-target distance on the template heated up to $600{ }^{\circ} \mathrm{C}$ by a radiative heater. $\mathrm{Ar} / \mathrm{O}_{2}$ mixture with $1 / 1$ flow ratio is used as a sputter gas at total pressure 2 mTorr. It is shown below, that these conditions support formation of the BSTO films with low grain misorientation which, in turn, reduces the generation of shear waves leaking through the Bragg reflector. The Al top electrodes with Ti adhesion layer (not shown in the Fig. 1) are deposited by e-beam evaporation. A lift-off process is used to

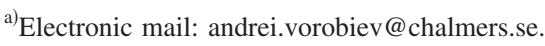

pattern the top electrodes in the form of central circular patches surrounded by cocentric outer electrodes. The relatively large area of the outer top electrodes provides effective $\mathrm{dc}$ and ac connection to the bottom electrode. Sets of the TFBAR test structures with $200 \mu \mathrm{m}$ diameter of the outer electrodes and different diameters of the central electrodes varying in the range $20-60 \mu \mathrm{m}$ are fabricated and characterized.

The complex input impedance $Z=\operatorname{Re} Z+j \operatorname{Im} Z$ and admittance of the test structures are calculated using $S_{11}$ parameters measured by an Agilent N5230A vector network analyzer and GSG microprobes in the frequency range 1-10 GHz. The modified Butterworth-Van Dyke (MBVD) circuit model (Ref. 8) is used for de-embedding of the electrical loss associated with series resistance $R_{s}$ composed by the ring section of the Pt bottom electrode and contact resistance between $\mathrm{Al}$ pads and probe tips. The series resistance in the MBVD model (Ref. 8) is found as the real part of impedance at the high frequency limit which allows us to separate it from the equivalent resistance of BSTO capacitor associated with other non-ohmic contributions. The series resonance

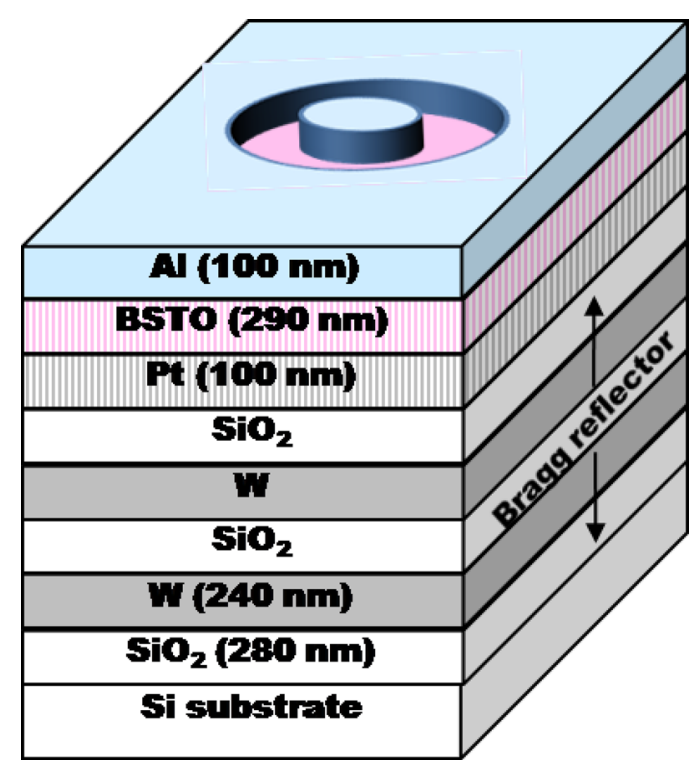

FIG. 1. (Color online) Three-dimensional schematic image of a TFBAR test structure. 


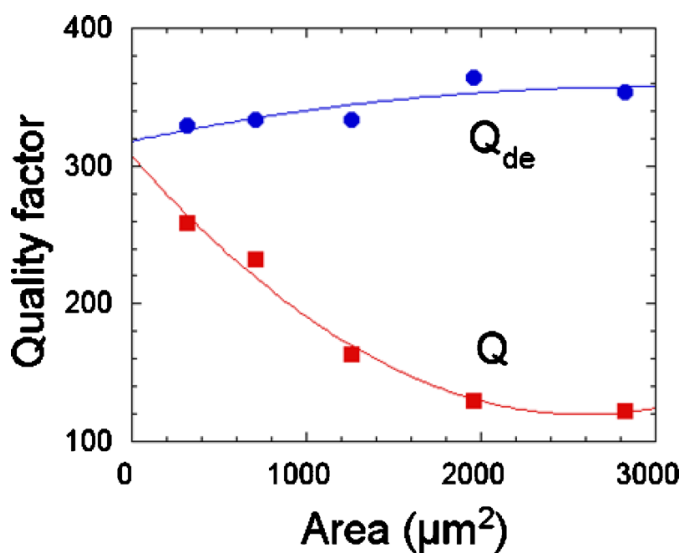

FIG. 2. (Color online) Measured and de-embedded $Q$-factors vs electrode area at $25 \mathrm{~V} \mathrm{dc}$ bias. The lines are polynomial curve fit.

frequency $f_{s}$ is defined as frequency of maximum of the real part of the admittance. The $Q$-factors are calculated as follows:

$$
Q=\left.\frac{1}{2} f_{s} \frac{\partial \varphi}{\partial f}\right|_{f=f_{s}},
$$

where the phase angle $\varphi=\operatorname{arctg}(\operatorname{Im} Z / \operatorname{Re} Z)$ is for the device under test and $\varphi=\operatorname{arctg}\left[\operatorname{Im} Z /\left(\operatorname{Re} Z-R_{s}\right)\right]$ for the deembedded $Q$-factor which includes other non-ohmic losses. Microstructure of the BSTO films is analyzed using X-ray diffraction (XRD) and atomic force microscopy (AFM) techniques. The XRD spectra are obtained using a Philips X'pert SW 3040 diffractometer equipped with a point $\mathrm{Cu} K \alpha$ radiation source, an MRD lens, a thin collimator, and a Ni filter. The BSTO film surface is analyzed using a Digital Instruments Dimension 3000 SPM operating in tapping mode. The AFM images are analyzed using an SPIP software tool.

Figure 2 shows the measured and de-embedded $Q$-factors versus area of the test structure electrode at $25 \mathrm{~V}$ dc bias. The $Q_{\mathrm{de}}$ increases slightly with area since the relative mechanical loading by the probe tip decreases. The large difference between $Q_{\mathrm{de}}$ and $Q$ for large electrode areas indicates the significant contribution of the series resistance loss in the total loss balance, firs of all, due to relatively long path of the ring section of the Pt bottom electrode. The $Q$ increases and approaches $Q_{\mathrm{de}}$ when the electrode area decreases since the motional resistance of the small area TFBARs dominates over the series resistance. ${ }^{8}$ In particular, this validates the used method of the $R_{s}$ de-embedding.

Figure 3 shows the series resonance frequency and deembedded $Q$-factor of a TFBAR test structure with $60 \mu \mathrm{m}$ diameter central electrode versus dc bias voltage. The $Q_{\mathrm{de}}$ approaches zero at low fields because of the nature of the field induced piezoeffect. The de-embedded product $Q_{\text {de }} f$ $=1875 \mathrm{GHz}$ is almost three times higher than the highest reported value $700 \mathrm{GHz}$ for the BSTO SMR (Ref. 4) and close to that required for the filter applications $2000 \mathrm{GHz} .^{2}$ The $Q f=1330 \mathrm{GHz}$ product of the device under test with diameter $20 \mu \mathrm{m}$ (Fig. 2) is more than three times higher than the highest reported value $420 \mathrm{GHz}$ for the BSTO SMR. ${ }^{1}$ We explain the improvement in the $Q$-factor by reduction in the BSTO film microstructure imperfections. The latter is responsible for generation of leaking waves. Usually, in TFBARs the following three main leaking wave mecha-

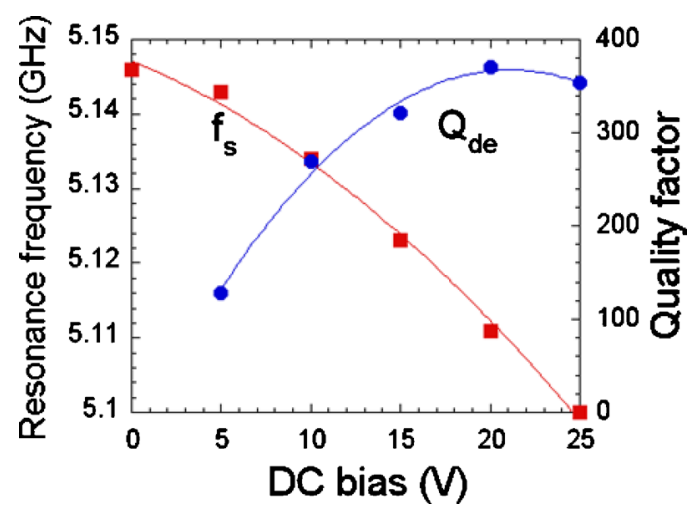

FIG. 3. (Color online) Series resonance frequency and de-embedded $Q$-factor of a TFBAR vs dc bias voltage. The lines are polynomial curve fit.

nisms are considered: (i) laterally leaking waves; (ii) waves scattered by surface roughness; and (iii) waves leaking through the Bragg reflector. ${ }^{2}$ Experiments have shown that lateral wave leakage is not a dominant loss mechanism in SMRs operating in the $Q$-regime up to $2500 .^{2}$ Analysis of the AFM images of our BSTO films indicates that the rms of the surface roughness increases slightly in the range $2.5-3.5 \mathrm{~nm}$ with increasing the BSTO film growth temperature while the $Q_{\mathrm{de}}$ increases rapidly from 130 to 370 . Thus, we conclude that the improvement in the $Q$-factor cannot be associated with reduction in scattering by the surface roughness. The generation of shear waves leaking through the $\lambda / 4$ Bragg reflector into the substrate is a loss mechanism specific for SMRs using longitudinal acoustic mode. ${ }^{2,9}$ It is shown that a piezoelectric thin film with mean/net tilt of the crystalline $c$-axis (rocking curve offset) generates the shear waves. ${ }^{10,11}$ Further, we assume that each individual grain of a piezoelectric film, if tilted, produces a local shear wave as well. Thus, apparently, a requirement for reduction in shear wave loss is fabrication of piezoelectric film with low rocking curve and low rocking curve offset. The condition for low mean tilt is a symmetric distribution of the deposition flux. ${ }^{11}$ The condition for low rocking curve is high mobility of adatoms which can be achieved by deposition at high temperature and/or gas pressure low enough to prevent thermalization of the sputtered species. ${ }^{12}$ We have established these conditions in our deposition system using on-axis configuration and extremely low gas pressure 2 mTorr. The on-axis deposition provides symmetric net flux and, hence, low mean tilt of the grain $c$-axis. Additionally, a template in on-axis position of a magnetron sputtering system is, usually, subjected to intensive irradiation by secondary electrons which are not trapped by magnetic field. This may result in significant increase in effective temperature on the condensation surface. The mean free path of the sputtered species at gas pressure of $2 \mathrm{mTorr}$ and temperature $600{ }^{\circ} \mathrm{C}$ is approximately $10 \mathrm{~cm}$ which means there is no thermalization. For comparison, the BSTO films in the TFBARs with highest so far reported product $Q_{\text {de }} f=700 \mathrm{GHz}$ is deposited at 25 mTorr. ${ }^{4}$ The XRD spectra of our TFBARs test structures reveal no BSTO peaks other than $\{111\}$ which in turn (we assume) is masked by strong Pt $\{111\}$ and $\mathrm{W}\{111\}$ peaks. This does not allow measurements of the BSTO grain rocking curve by means of XRD. We plan to use high resolution transmission electron microscopy cross section selected area diffraction analysis to measure the local grain misalignment and establish its correla- 
tions with the deposition conditions and the TFBARs acoustic performance.

In conclusion, BSTO TFBARs with improved product $Q f=1330 \mathrm{GHz}$ (de-embedded $Q_{\text {de }} f=1875 \mathrm{GHz}$ ) have been demonstrated. The improvement is achieved by deposition of the BSTO films in on-axis configuration at extremely low pressure 2 mTorr. These conditions support formation of the BSTO films with low grain misorientation which, in turn, reduces the generation of shear waves leaking through the Bragg reflector and the associated acoustic loss.

This work was supported by the project VR FBAR (2009-3460) of the Swedish Research Council.

${ }^{1}$ J. Berge, M. Norling, A. Vorobiev, and S. Gevorgian, J. Appl. Phys. 103, 064508 (2008).

${ }^{2}$ K.-Y. Hashimoto, RF Bulk Acoustic Wave Filters for Communications
(Artech House, Norwood, 2009).

${ }^{3}$ A. Noeth, T. Yamada, P. Muralt, A. K. Tagantsev, and N. Setter, IEEE Trans. Ultrason. Ferroelectr. Freq. Control 57, 379 (2010).

${ }^{4}$ G. N. Saddik, D. S. Boesch, S. Stemmer, and R. York, IEEE MTT-S Int. Microwave Symp. Dig. 2008, 1263.

${ }^{5}$ X. Zhu, J. D. Phillips, and A. Mortazawi, IEEE MTT-S Int. Microwave Symp. Dig. 2007, 671.

${ }^{6}$ R. Nava, R. Callarotti, H. Ceva, and A. Martinet, Phys. Rev. 188, 1456 (1969).

${ }^{7}$ J. F. Rosenbaum, Bulk Acoustic Wave Theory and Devices (Artech House, Norwood, 1988).

${ }^{8}$ M. Norling, J. Berge, and S. Gevorgian, IEEE MTT-S Int. Microwave Symp. Dig. 2009, 101.

${ }^{9}$ J. Kaitila, Proc.-IEEE Ultrason. Symp. 2007, 120.

${ }^{10}$ F. Martin, M.-E. Jan, B. Belgacem, M.-A. Dubois, and P. Muralt, Thin Solid Films 514, 341 (2006).

${ }^{11}$ J. Bjurström, G. Wingqvist, and I. Katardjiev, Proc.-IEEE Ultrason. Symp. 2005, 321.

${ }^{12}$ G. F. Iriarte, F. Engelmark, and I. V. Katardjiev, J. Mater. Res. 17, 1469 (2002) 